Similarly, (3 10546 ), (4 756 10), and (4 1065 7) follow by applying first (5) and then (1).

Transforming (3 33333 ) by $S^{6}$ and replacing $S^{8} T S^{3} T$ by its inverse $T S^{8} T S^{3}$, given by (4), we get

$$
T S^{8} T S^{6} T S^{3} T S^{3} T S^{6}=I,
$$

a relation following by taking the inverse of ( 885535 ).

Transforming (7 7777 ) by $S^{2}$ and replacing $S^{5} T S^{7} T$ by its inverse $T S^{4} T S^{6}$, given by $(5)$, we get

$$
T S^{4} T S^{2} T S^{7} T S^{7} T S^{2}=I,
$$

a relation following by taking the inverse of (4 4979 ).

Hence the simple group of order 660 is generated by two operators $S$ and $T$ subject to relations (1), (4), and (5).

The University of Chicago,

$$
\text { October 22, } 1902 .
$$

\title{
THE CARLSBAD MEETING OF THE DEUTSCHE MATHEMATIKER-VEREINIGUNG, SEPTEMBER, 1902.
}

THE annual meeting of the Deutsche Mathematiker-Vereinigung was held at Carlsbad, September 21-26, forming as usual a part of the Versammlung Deutscher Naturforscher und Aertze. At the business meeting of the Society, committees were appointed to make arrangements for the international mathematical congress which will be held at Heidelberg in August, 1904. A committee was also appointed to consider the feasibility of forming at some point in Germany a very complete collection of mathematical literature. Professor Gutzmer resigned from the position of secretary of the society, and Professor Krazer was elected his successor. The meeting of the Society will be held next year at Cassel.

The following papers were read at the meeting:

Professor Czuber, Vienna: "A theorem in the theory of errors, and its application."

Professor GRÜNWALD, Prague: "Derivatives with variable index as analytic functions of the latter, and a related functional operation." 
Professor Haskell, California : "The representation of certain resultants as determinants."

Dr. JAHNke, Berlin : "An elementary theory of the theta functions of one and two arguments."

Dr. JAHNkE, Berlin : "The relation of Steiner to Jacobi."

Professors KLeIN, Göttingen, and MEYer, Königsberg: "The progress on the Encyklopädie der mathematischen Wissenschaften."

Professor KoHn, Vienna : "On a certain sextuple of rays."

Professor Kowalewski, Greifswald : "A report on Lie's theory of transformation groups."

Professor Kowalewski, Greifswald : "On a criterion of du Bois-Reymond for the development in Fourier series."

Professor Kow Alewski, Greifswald: "A new characteristic of the projective group of a norm curve."

Dr. Ltebmann, Leipsic : "On the conformal representation of convex surfaces upon the sphere."

Professor MEYER, Königsberg: "On the spheres inscribed in a tetrahedron."

Professor E. MüLlen, Vienna: "On the theory of linear systems of curves and surfaces of the second order."

Professor F. MÚller, Steglitz: "The abbreviations of the titles of mathematical publications."

Professor Schubert, Hamburg : "Enumerative relations for incidence and coincidence in linear space of higher dimensions."

Dr. Steinitz, Berlin : "A report on polyhedra."

Dr. von Sterneck, Vienna: "On an analogy to the additive number theory."

Professor Ẅ̈Lsch, Brünn : "On binary analysis."

Professor Czuber developed the relations which hold for a linear form of $n$ independent errors of observation, when the errors follow singly the exponential law, and applied the results to direct observations of equal exactness.

Professor Grünwald spoke on his researches in the theory of the derivatives with variable index, and their development in powers of the latter. The coefficients in the development are found by linear functional operations, and are represented by definite integrals. The application to the solution of certain linear differential equations was mentioned. 
Sylvester's dialytic method, applied to finding the resultant of forms of more than two variables, gives a determinant of too high an order - the resultant with a foreign factor. Cayley has found the resultant in the form of a determinant without foreign factor for the case of three ternary quadratic forms, and Hesse for the case of three ternary forms of which two are quadratic and one linear, and of four quaternary forms of which three are quadratic and one linear. These results follow (those of Hesse less directly) from the theorem, that if $n$ homogeneous forms in $n$ variables vanish simultaneously, then the Jacobian vanishes, and if the forms are of the same degree, the first derivatives of the Jacobian vanish also. Professor Haskell adds to this theorem that, if the forms are of the same degree, the second derivatives of the Jacobian are proportional to certain linear functions of the second derivatives of the forms, and the application of this theorem gives the resultant of four or of five quadratic forms, homogeneous in four or five variables respectively, in the form of a determinant without foreign factor.

Dr. Jahnke spoke on Caspary's theory of the theta functions and gave some further developments. In Jacobi's lectures the theory of the theta functions was developed from their definition by series of exponential functions. Caspary used this definition but gave the theory a purely algebraic character without function-theoretical considerations, introducing the orthogonal system with Euler's form for the coefficients in order to express the many algebraic relations between the thetas. For further development of the theory only the algebraic and differential identities between the elements of an orthogonal system are required. The introduction to the elliptic transcendentals, as given by Caspary, appears elementary enough for the use of students of physics and technology.

In his second paper Dr. Jahnke read some recently discovered letters of Steiner and Jacobi.

Professors Klein and Meyer spoke on the progress of the Encyklopädie. The last part of Volume I containing the index will probably appear before Easter. The chief difficulty in Volume II has been overcome, but further publication will not be immediate. Volume III, on geometry, is divided into three 
main parts, and articles from each part will appear in the next few months ; in Part I, Enriques, Principles of Geometry ; in Part II, Dingeldey, Conic Sections; in Part III (Differential Geometry), Mangold, Space Curves and Surfaces. Volume IV (Mechanics) is progressing satisfactorily. Publication will soon begin on Volume V (Physics), with Bryan's article on Thermodynamics, and also on Volume VI (Geodesy and Geophysics).

Professor Kohn's paper is in abstract as follows: There is a single system of six lines, such that every interchange is produced by a collineation or correlation. These lines may be called an equianharmonic ray sextuple from the fact that the four points of intersection of every line that meets four of them are equianharmonic. If an icosahedron form be interpreted on a cubic space curve the six chords given by the six "pairs" of the icosahedron form are such a system; the six corresponding axes form a second system which is related to the first in many ways. Interesting results follow from the fact that two surfaces of second order, one containing three of the lines of the system, the other the remaining three, cut each other in the edges of a skew four-edge. Corresponding to the ten cases where the six lines reduce to two sets of three, are ten tetrahedra, each of which is inscribed in and circumscribed about every other. The forty vertices and forty sides form a Witting configuration, and it follows that the Klein group of 6 ! collineations is a subgroup of the hyperelliptic collineation group under which the Witting configuration is invariant. The above is in close relation to results of Klein, Witting, Maschke, and Burkhardt.

Professor Kowalewski's first paper was a report on Lie's theory of transformation groups. Much of Lie's work has a value apart from his group theory, e.g., the idea of element and element union, important in the theory of differential equations; that of contact transformations, which Lie conceived in its generality in the first years of his activity ; and, of greatest importance, the infinitesimal transformation. Lie was in possession of most of these ideas in 1873 when he began to develop his theory of transformation groups, above all, however, that of the infinitesimal transformation. He had found in 1872 that an ordinary differential equation of first order with a known 
infinitesimal transformation could be integrated by quadrature, and had proposed to himself the problem : Of what value for the integration of a system of differential equations is the knowledge of infinitesimal transformations which leave the system unaltered? He considered the case of a complete system of linear partial differential equations in detail. If two infinitesimal transformations $X f$ and $Y f$ leave such a system unchanged, then this holds also for the "Klammerausdruck"

$$
X(Y f)-Y(X f)=(X Y) .
$$

By this theorem the question is reduced to the consideration of a complete system, with $r$ known infinitesimal transformations $X_{1} f_{1}, \cdots, X_{r} f_{r}$ which satisfy relations of the form

$$
\left(X_{i} X_{k}\right)=\sum C_{i k s} X_{s} f
$$

and these relations hold for all point transformations. This was the starting point for the theory of transformation groups. Lie found by involved reckoning that $r$ independent infinitesimal transformations which satisfy relations of the above form, belong to a continuous group with $r$ parameters, and showed conversely that every such group contains $r$ independent infinitesimal transformations which satisfy the above relations. $\mathrm{He}$ had then, although without rigorous proofs, the two fundamental theorems of his group theory. The first presentation of Lie's results is in an article by Klein and Lie in the Göttinger Nachrichten, December 3, 1874. The differential equations of his later first fundamental theorem occur here, while the third, which relates to the constants $C_{i k s, s}$, appeared in 1876 in the Norwegian Archiv. In the Göttingen note Lie indicates some applications of his theory to ordinary differential equations, which he did not complete until 1882 (Archiv for Mathematik, volumes 8 and 9). In 1882 Lie applied his theory of finite groups to infinite groups by considering only such groups as can be defined by differential equations. He showed also that every such group determines infinitely many differential invariants (Mathematische Annalen, volume 24).

A norm curve in $R_{n}, i . e$. , a rational curve of order $n$ which is contained in no plane point manifold of $R_{n}$, admits a group of $\infty^{3}$ projective transformations. Professor Kowalewski, in his second paper, gave a complete enumeration of all projective transformation groups which contain the above as a subgroup. 
For $n>2$ there exists in general (except for the group of all projective transformations) only one such group. The single exception is in case of $R_{6}$, where a fourteen group exists, which has the Killing constitution and plays a part in the work of Engel.

In his third paper, Professor Kowalewski calls attention to a little known criterion for the convergence of a Fourier series which was published by P. du Bois-Reymond in 1881 in the Comptes Rendus and earlier in Crelle. A simpler proof of this criterion was indicated.

Closely related to the theorem that a closed convex surface can not in general be deformed (verbogen), without introducing singularities, is the following theorem: A closed convex surface can not in general be conformally represented, without singularities, upon the sphere. Dr. Liebmann considered a special surface of revolution which admits at most a three parameter group of regular conformal transformations, while for the sphere the six parameter group of homographic transformations forms such a regular conformal group. The surface cannot, therefore, be represented conformally upon the sphere without singularities, and the theorem holds for this special surface. Other such examples can be found showing that the theorem in general holds.

A theorem of Schwarz states that if on each face of a tetrahedron circumscribing a sphere lines are drawn from the point of tangency to the vertices, then the corresponding angles about the points of tangency on the four faces are equal. Interpreting this theorem by projective geometry, Professor Meyer gave some interesting generalizations.

Projective geometry is generalized after Klein by considering the properties of geometrical configurations invariant under a more general transformation group. Of special importance is the projective geometry of curves and surfaces of the second order. A simple theorem of this geometry may contain many results from the standpoint of the ordinary projective geometry. Professor E. Müller showed this by applying the theorem, that in a web of conics (Kegelschnittgewebe) two families always have a common conic, to two special webs, 
first, one with a double point, and second, one whose curves are tangent to two lines. Three conics $\phi_{1}, \phi_{2}, \phi_{3}$, of which $\phi_{3}$ has double tangency with $\phi_{1}$, determine a web of the first kind, and from the theorem follows that in the family $\left(\phi_{3}, \phi_{2}\right)$ there are three conics each of which passes through a pair of opposite vertices of the tangential complete quadrilateral common to $\phi_{1}$ and $\phi_{2}$. The theorem of Darboux follows when $\phi_{3}$ is a point pair, and theorems of Chasles on confocal conics result through metric specialization. 'The application of the above general theorem to the second web also gives interesting theorems, e.g., the point pairs of two projective point rows of the plane belong to a web of this kind. The fruitfulness of similar considerations for higher dimensions was indicated.

Professor F. Müller spoke on the abbreviations of the titles of mathematical journals, and presented a list of some 600 proposed abbreviations. This list will be manifolded for the present, and eventually published.

In the enumerative geometry only those incidence formulæ have been generalized to linear space of $n$ dimensions, by Schubert, Pieri and others, which relate to a line and incident point. Professor Schubert now attacks the general problem, and finds all the fundamental incidence and coincidence formulæ for two linear spaces of dimensions $n$ and $n+m$.

Dr. Steinitz gave a report on the general theory of the polyhedron, starting from the results of Euler. The symbolic table of Catalan, the work of Eberhardt on polyhedron " fields" and the general question of polyhedra of multiple connectivity were treated at some length.

In the additive number theory the number of ways is sought in which a given number can be formed by the addition of given elements. An analogous question is the following: If $n$ and $M$ are given numbers, in how many ways can a number congruent to $n(\bmod . M)$ be formed additively from the elements $1,2, \ldots$, $M-1$. Stern (1863) studied the question for prime moduli. In the paper of Dr. von Sterneck the general problem was considered, with following results: If $(n)_{i}^{0}$ is the number of such representations of $n(\bmod . M)$ by $i$ elements, and $(n)_{i}$ the similar number when zero may appear as an element, then 


$$
\begin{gathered}
(n)_{i}^{0}=(n)_{i}-(n)_{i-1}+(n)_{i-2}-\cdots+(-1)^{i}(n)_{0}, \\
i(n)_{i}=\sum(n-e)_{i-1}-\sum(n-2 e)_{i-2}+\cdots+(-1)^{i} \sum(n-i e)_{0} .
\end{gathered}
$$

In the summations $e$ assumes all values of a complete remainder system with respect to $M$, and $(n)_{0}=0$ except when $n \equiv 0$ $(\bmod . M)$, in which case $(n)_{0}=1$.

The difference between the numbers of even and odd representations of $n$ is then

$$
\Delta(n)=\sum(-1)^{i}(n)_{i}^{0}
$$

In particular, for $M \equiv p^{k}$,

$$
\Delta(n)=0, \quad-p^{k-i}, \quad p^{k}-p^{k-i}
$$

according as $n$ is not divisible by $p^{k-i}$, is divisible by $p^{k-i}$, or is divisible by $p^{k}$.

For any $M=p_{1}^{\alpha_{1}} p_{2}^{\alpha_{2}} \cdots p_{k_{k}}^{\alpha_{k}}$ the theorem holds: If $n$ contains any $p_{i}$ to a power $\leqq a_{i}-2$, then $\Delta(n)=0$. If $n$ contains the factors $p_{1}, p_{2}, \cdots, p_{i}$ to the powers $a_{1}-1, a_{2}-1, \cdots, a_{i}-1$, and the factors $p_{i+1}, \cdots, p_{k}$ to the powers $a_{i+1}, \cdots, a_{k}$, then

$$
\Delta(n)=(-1)^{i} \frac{\phi(M)}{\left(p_{1}-1\right)\left(p_{2}-1\right) \cdots\left(p_{i}-1\right)} .
$$

If $n$ contains all the factors of $M$ to the same degree, then $\Delta(n)=\phi(M) . \quad \phi(M)$ is the Euler function.

Further relations can be developed from the theorem : The difference $\psi(M)$ between the number of odd and even representations when the H. C. F. of the elements is relative prime to the modulus, satisfies the equation $\sum \psi(d)=\phi(M)$, where $d$ takes on the values of all factors of $M$, and is then the number theory derivative of the Euler function $\phi(M)$.

Professor Wälsch spoke on the application of the theory of binary forms to geometry and mechanics. By the introduction of the Kugelkreis, points, lines, and planes are represented by binary forms. A point $a$ lies in a plane $b$ when the second "Ueberschiebung" of the form $a$ with $b$ is unity. A vector can also be represented by a binary form, and the first and second "Ueberschiebungen" of two forms give the two vector products. A rational space curve of order $n$ is given by the form $r_{y}^{n} s_{x}^{2} / c_{y}^{n}$. In applying the binary form to mechanics, the 
expressions for the ellipse of inertia, kinetic energy, and the equations of motion, take very simple forms.

I wish to thank the speakers for the use of manuscripts, and to express my indebtedness to many who gave me abstracts of their papers.

GöTTINGEN,

C. M. MASON.

October, 1902.

\section{SHORTER NOTICES.}

Compte rendu du deuxième Congrès international des Mathématiciens tenu à Paris du 6 au 12 aoút 1900. Procès-verbaux et Communications publiés par E. Duporcq. Paris, GauthierVillars, 1902. 8vo. $455 \mathrm{pp}$.

THE members of the second international congress of mathematicians may have felt a little annoyance at the delay in the publication of the official report; but now that this is at last in their hands, the delay no longer appears un reasonable. A glance through the volume makes it plain that the editor's task has been very serious, inasmuch as its execution has depended on the good pleasure of so many contributors, and these so widely dispersed, that it has been quite beyond his power to expedite matters. Editor and publisher are to be congratulated on the beautiful volume which has recently appeared; it was worth waiting for.

The first 26 pages contain the minutes of the sectional and general meetings; pages 27-153 are occupied by the four principal addresses, with which is placed also a French translation of Hilbert's survey of the future problems of mathematics; pages 154-450 are devoted to the sectional papers. Of these thirty-two are given, thus leaving six or eight unreported for reasons beyond the editor's control. In general the papers appear in a French version, the exceptions being one in Italian and eight in English. As a matter of fact, the majority of the communications were made in French, but there were certainly some in German.

The most interesting articles for present reading are naturally those of a somewhat general character, for the results of more special interest have not been held back pending this publica- 